\title{
Introduction to Breast Disease Review Issue on Metastasis
}

\author{
Lalage Wakefield and Kent Hunter \\ National Cancer Institute, National Institutes of Health, Bethesda, MD, USA
}

Metastasis is the most dreaded aspect of the carcinogenic process. More than $90 \%$ of all cancer deaths are attributable to the consequences of the primary tumor successfully colonizing distant organs. Unlike the situation with colon cancer, a patient with breast cancer can never be considered "cured", since as many as a third of breast cancer patients who have apparently curative surgery for their primary tumors ultimately relapse with metastatic disease, sometimes decades later. Much effort is now devoted to understanding this process of metastasis, and finding ways to predict and prevent its occurrence. This issue covers recent advances in the field, specifically as they relate to breast cancer.

The availability of new tools and technological approaches has prompted a reconsideration of the very definition of a metastasis. In the first article of this issue, Danny Welch explodes a number of commonly held myths and proposes a new definition of a metastasis, with important implications for clinical staging. Surgical autopsies are performed much less frequently these days, so few pathologists now have the opportunity to probe the natural history of the metastastic process in humans as extensively as in the past. In the second article, David Tarin brings insights from his 30-year experience, as surgical breast cancer pathologist and breast cancer researcher, to place recent advances in cellular and molecular research in the context of what is known about the pathogenesis of breast cancer metastasis. Sui Huang and Donald Ingber take the field beyond conventional gene-centered explanations of cancer progression, by presenting a novel conceptual framework for cancer progression based on the system-level dynamics of regulatory networks. They describe mathematically and biologically how mutational rewiring of these networks can trap cancer cells in "embryonic attractors" on an epigenetic attractor landscape, stabilizing system states with emergent properties that would tend to promote metastasis.

Primary tumors and metastases must adapt to a changing and frequently hostile microenvironment. Naz Chaudary and Richard Hill address the role of local hypoxia, a feature of the majority of breast tumors, in promoting the metastatic phenotype. David Lyden and coworkers discuss their recent work suggesting that the primary tumor can direct the establishment of a "premetastatic niche" in a distant tissue, such that it provides a receptive microenvironment for the disseminated tumor cell, and Antoine Karnoub and Robert Weinberg review the role of chemokines in mediating some of the evolving transactional processes between tumor and stroma.

Only a small minority of tumor cells leaving the primary site go on to successfully establish distant metastases. Understanding the nature of this successful minority and the conditions necessary for their outgrowth will be critical for effective treatment. Ann Chambers and colleagues address the implications of the cancer stem cell hypothesis in understanding the phenomenon of metastatic dormancy. Yael Goldfarb and Shamgar Ben-Eliyahu discuss evidence that surgery to remove the primary tumor can actually accelerate establishment or further development of metastases, through transient suppression of cell-mediated immunity. They discuss modifications of surgical procedures and perioperative support that could reduce this undesirable effect of surgery. In a related vein, Wei-Zen Wei and colleagues explore the prospects for controlling breast cancer metastasis by active immunization against tumor antigens. 
It has long been known that breast cancer metastasizes most commonly to only a few organs, particularly lung, liver, bone. Yibin Kang describes the application of functional genomics and in vivo imaging to address the molecular mechanisms that underlie these observed tissue tropisms. Brain metastases used to be relatively rare in breast cancer patients, but are becoming much more prevalent with the successful use of Herceptin to control residual disease below the neck in patients with aggressive HER2/ErbB2 positive tumors. The underlying biology and unique set of therapeutic challenges posed by metastasis to this site are discussed by Pat Steeg and coworkers.

Global gene expression profiling of breast tumors in large patient cohorts with outcome data has lead to the exciting finding that metastatic tumors have different expression profiles from non-metastatic tumors, as discussed by Dimitry Nuyten and Marc van de Vijver. The utility of gene expression signatures to stratify patients according to their relative risk of developing metastasis is currently being tested in clinical trials, with the hope that aggressive treatment can be applied more selectively. Intriguingly, the work of Kent Hunter and colleagues suggests that the "metastatic signature" may reflect constitutional genetic polymorphisms rather than tumor-specific changes in gene expression, raising the possibility of identifying individuals who are genetically at high risk for developing metastases prior to disease development. Ultimately identification of the molecular mechanisms underlying metastasis susceptibility could lead to novel strategies for preventing breast cancer metastasis in at-risk populations, and remove the dreaded spectre of this killer aspect of the disease. 